\title{
Article \\ Pseudococyclic Partial Hadamard Matrices over Latin Rectangles
}

\author{
Raúl M. Falcón*(D), Víctor Álvarez (D), María Dolores Frau (D), Félix Gudiel (D) and María Belén Güemes \\ Department of Applied Mathematics I, University of Seville, 41004 Sevilla, Spain; valvarez@us.es (V.Á.); \\ mdfrau@us.es (M.D.F.); gudiel@us.es (F.G.); bguemes@us.es (M.B.G.) \\ * Correspondence: rafalgan@us.es
}

\begin{abstract}
The classical design of cocyclic Hadamard matrices has recently been generalized by means of both the notions of the cocycle of Hadamard matrices over Latin rectangles and the pseudococycle of Hadamard matrices over quasigroups. This paper delves into this topic by introducing the concept of the pseudococycle of a partial Hadamard matrix over a Latin rectangle, whose fundamentals are comprehensively studied and illustrated.
\end{abstract}

Keywords: Hadamard matrix; Latin rectangle; pseudocoboundary; pseudococycle; quasigroup

MSC: 05B20; 05B15; 20N05

\section{Introduction}

A (binary) Hadamard matrix is a square matrix $H$ of order $n$ with entries in the set $\{-1,1\}$ such that $H H^{t}=n I_{n}$. As such, all its rows (equivalently, columns) are pairwise orthogonal, and hence, its order must be 1,2, or a multiple of 4 . The Hadamard conjecture [1] ensures the existence of Hadamard matrices for every order multiple of 4. It has remained open for more than a century [2].

In 1993, as a new way for generating combinatorial designs that generalizes the group

Citation: Falcón, R.M.; Álvarez, V.;

Falcón, M.D.; Gudiel, F.;

Güemes, M.B. Pseudococyclic Partial Hadamard Matrices over Latin

Rectangles. Mathematics 2021, 9, 113.

http://doi.org/10.3390/math9020113

Received: 2 December 2020

Accepted: 4 January 2021

Published: 6 January 2021

Publisher's Note: MDPI stays neutral with regard to jurisdictional clai$\mathrm{ms}$ in published maps and institutional affiliations.

Copyright: $\odot 2021$ by the authors. Licensee MDPI, Basel, Switzerland. This article is an open access article distributed under the terms and conditions of the Creative Commons Attribution (CC BY) license (https:// creativecommons.org/licenses/by/ $4.0 /)$. development method, in combinatorial design theory, Horadam and de Launey [3] (see also $[4,5])$ introduced the fundamentals of the so-called cocyclic development over finite groups. In this context, a matrix with entries in the set $\{-1,1\}$ is said to be cocyclic over a finite group $(G, \cdot)$ if there exists a map $\phi: G \times G \rightarrow\{-1,1\}$ satisfying the so-called cocycle equation:

$$
\phi(i \cdot j, k) \phi(i, j) \phi(j, k) \phi(i, j \cdot k)=1,
$$

for all $i, j, k \in G$, so that the matrix under consideration is Hadamard equivalent to the cocyclic matrix $M_{\phi}:=(\phi(i, j))_{i, j \in G}$. That is, they are equal up to permutation or negation of rows and columns. The map $\phi$ is a cocycle $[3,6]$ over the group. A cocyclic matrix necessarily has a constant row and a constant column. According to the cocyclic test [6], it is Hadamard whenever the summation of all the entries of each row is zero, except for the ones in its constant row. As such, determining whether a cocyclic matrix is Hadamard is computationally much faster than checking the definition of a Hadamard matrix.

In 1995, Horadam and de Launey [6] proved that this cocyclic framework provides an excellent structural approach for dealing with the Hadamard conjecture, which would be a consequence of the so-called cocyclic Hadamard conjecture [3], for which a cocyclic Hadamard matrix of order $4 t$ exists for every positive integer $t$. It is so that many known families of Hadamard matrices are cocyclic over certain groups: Sylvester matrices [7], Paley matrices [1], Williamson matrices [8], or Ito's type Q matrices [9] (see also [2,10-14] for some constructions in this regard). Nevertheless, the cocyclic framework turned out to fail [12] for two of the most prolific families of Hadamard matrices: the two-circulant core Hadamard matrices [15] and the Goethals-Seidel arrays [16]. 
Very recently, a new approach introduced by the authors of [17] has successfully dealt with a cocyclic development of Goethals-Seidel arrays, not over a group, but over a family of Moufang loops. This approach is comprehended in the new theory of cocyclic development over quasigroups and Latin rectangles, which has also recently been introduced by the authors in [18]. More specifically, a cocycle $\phi$ over a quasigroup $(Q, \cdot)$ is a map $\phi: Q \times Q \rightarrow\{-1,1\}$ satisfying the cocycle Equation (1) for all $i, j, k \in Q$. If an ordering of the elements of $Q$ is established, then the cocycle $\phi$ is uniquely represented by the cocyclic matrix $M_{\phi}:=(\phi(i, j))_{i, j \in Q}$. In particular, the quasigroup $(Q, \cdot)$ must be a loop whenever the matrix $M_{\phi}$ is Hadamard. Moreover, the cocyclic Hadamard test also holds in this case.

The main aspect of this new approach is the fact that associativity is no longer a necessary condition for dealing with any of the concepts and results that are usually involved in the cocyclic development over finite groups. It is so thatthe existence of coboundaries over non-associative loops has already been proved [17]. In this regard, we remind the reader that a cocycle $\phi$ over a quasigroup $(Q, \cdot)$ is called a coboundary if there exists a map $\partial: Q \rightarrow\{-1,1\}$ such that

$$
\phi(i, j)=\partial(i) \partial(j) \partial(i j), \text { for all } i, j \in Q \text {. }
$$

This coboundary $\phi$ is said to be elementary if there exists an element $h \in Q$ such that $\partial=\partial_{h}$, where $\partial_{h}(i)=-1$, if $i=h$, and $\partial_{h}(i)=1$ otherwise. From the cocycle equation, it is equivalent to say that

$$
i(j k)=h \Leftrightarrow(i j) k=h,
$$

holds for all $i, j, k \in Q$. It is straightforwardly satisfied in the case where $(Q, \cdot)$ is a group. Moreover, the cocycle equation has turned out not to be necessary in the quasigroup development theory. In this regard, a pseudocoboundary over the quasigroup $(Q, \cdot)$ is defined as any map $\psi_{h}: Q \times Q \rightarrow Q$ with $h \in Q$, satisfying Equation (2) for some $\partial_{h}$ described as above. By extension, a pseudococycle is any map $\psi=\left(\prod_{h \in H \subseteq Q} \psi_{h}\right) \phi$ that is obtained as the product of some pseudocoboundaries $\psi_{h}$ with $h \in H \subseteq Q$ and a cocycle $\phi$, all of them over a given quasigroup $(Q, \cdot)$. It is represented by the pseudococyclic matrix $M_{\psi}:=(\psi(i, j))_{i, j \in Q}$. If it is Hadamard equivalent to a given matrix, then the latter is called a pseudococyclic Hadamard matrix. Unlike the cocyclic framework over finite groups, every Goethals-Seidel array constitutes a pseudococyclic Hadamard matrix over a Moufang loop [17].

This last assertion corroborates the relevant role that non-associative quasigroups play in the generalization of the cocyclic framework over groups. This paper delves into this topic by focusing on the fundamentals of the pseudococyclic framework not only over quasigroups, but also over Latin rectangles. It enables us to generalize the classical notion of the cocycle of Hadamard matrices over groups to that of the pseudococycle of partial Hadamard matrices over Latin rectangles. We remind the reader in this regard that a partial Hadamard matrix is an $r \times n$ (binary) matrix $H$ with $r \leq n$ such that $H H^{t}=n I_{r}$. The recent implementation of these types of matrices in cryptography [19], experimental design [20], and quantum information [21] has awakened the interest in describing different ways of constructing them [22-25]. In addition, Latin rectangles may be implemented in Internet of Things (IoT) studies [26], coding theory [27,28], and modern 5G wireless networks [29]. Of particular interest in our study, the relevant role that quasigroups with few associative triples play in cryptography $[30,31]$ is remarkable. It is so that quasigroups with a high amount of non-associative triples are receiving particular attention [32-36].

The paper is organized as follows. In Section 2, we review some preliminary concepts and results on quasigroups and Latin rectangles that are used throughout the paper. In Section 3, we introduce and illustrate the notions of both the pseudocoboundary and pseudococycle over Latin rectangles. Then, we deal with the following two open problems concerning the pseudocoboundary framework over Latin rectangles. Both of them are completely answered in Section 4. 
Problem 1. Under which conditions may we ensure the existence of a partial Hadamard matrix that is a pseudocoboundary over a given Latin rectangle?

Problem 2. Under which conditions is a given partial Hadamard matrix a pseudocoboundary over a Latin rectangle?

We also deal with the problem of determining under which conditions we may ensure the existence of a partial Hadamard matrix that is pseudococyclic over a given Latin rectangle. In this regard, Sections 5 and 6 focus respectively on the pseudococyclic framework associated with trivial cocycles and the pseudococyclic framework related to non-trivial cocycles. Finally, since this paper has a high dependence on notation, a glossary of symbols is shown in Appendix A.

\section{Preliminaries}

Let us review some of the basic concepts and results on quasigroups and Latin rectangles that are used throughout the paper. We refer the reader to $[18,37]$ for more details about these topics.

A quasigroup [38] of order $n$ is a pair $(Q, \cdot)$ formed by a finite set $Q$ of $n$ elements that is endowed with a product - so that any two of the three elements $i, j, k \in Q$ in the equation $i \cdot j=k$ uniquely determine the third element. That is to say, the product $\cdot$ makes possible both the left and the right division in $Q$. A loop is a quasigroup $(Q, \cdot)$ with a unit element $e$ such that $i \cdot e=e \cdot i=i$ for all $i \in Q$. Every associative quasigroup is a group.

The Cayley table of a quasigroup of order $n$ constitutes a Latin square of the same order; that is, an $n \times n$ array with entries in a set of $n$ distinct symbols so that each symbol occurs exactly once per row and exactly once in each column. The removal of at least one row of a Latin square constitutes a Latin rectangle. More specifically, an $r \times n$ Latin rectangle, with $r \leq n$, is an $r \times n$ array with entries in a set of $n$ distinct symbols so that each symbol occurs exactly once per row and at most once in each column. From here on, let $\mathcal{R}_{r, n}$ denote the set of $r \times n$ Latin rectangles with entries in the set $[n]:=\{1, \ldots, n\}$. Further, $L[i, j]$ denotes the symbol contained in the cell $(i, j)$ of a Latin rectangle $L \in \mathcal{R}_{r, n}$.

Let $L \in \mathcal{R}_{r, n}$. If one defines the subset of symbols

$$
\mathcal{S}(L):=[r] \cup\{L[i, j] \mid 1 \leq i, j \leq r\} \subseteq[n],
$$

then a cocycle over $L$ is any function $\phi: \mathcal{S}(L) \times[n] \rightarrow\{-1,1\}$ satisfying the cocycle equation

$$
\phi(L[i, j], k) \phi(i, j) \phi(j, k) \phi(i, L[j, k])=1,
$$

for all positive integers $i, j \leq r$ and $k \leq n$. It is termed trivial if $\phi(i, j)=1$ for all $(i, j) \in \mathcal{S}(L) \times[n]$. Notice that the negation $-\phi$ of a cocycle $\phi$ over $L$ is also a cocycle over $L$. Further, every cocycle $\phi$ over $L$ is uniquely represented by the cocyclic matrix

$$
M_{\phi}:=(\phi(i, j))_{(i, j) \in \mathcal{S}(L) \times[n]} .
$$

The following example illustrates all these concepts. From here on, we represent, respectively, the symbols -1 and 1 in any given binary array with the symbols + and - .

Example 1. Let us consider the $2 \times 4$ Latin rectangle

$$
L \equiv \begin{array}{ll|l|l|}
1 & 2 & 4 & 3 \\
3 & 1 & 2 & 4 \\
\hline
\end{array}
$$

where we have highlighted those cells that are used to define the subset of symbols $\mathcal{S}(L)=\{1,2,3\}$. There exist exactly four cocycles over the Latin rectangle $L$ : the trivial one and the function $\phi: \mathcal{S}(L) \times[4] \rightarrow\{-1,1\}$, which are represented by the following matrix, together with their respective negations. 


$$
M_{\phi} \equiv \begin{array}{|c|c|c|c|}
\hline+ & + & + & + \\
\hline+ & + & + & - \\
\hline+ & + & - & + \\
\hline
\end{array}
$$

In particular, let us check that the function $\phi$ satisfies the cocycle Equation (3).

- If $i=1$, then $L[i, j]=j$ for all $j \in\{1,2\}$, and hence, the cocycle equation holds readily from the fact that the first row of the matrix $M_{\phi}$ is constant.

- If $(i, j)=(2,1)$, then $\phi(3, k) \phi(2,1) \phi(1, k) \phi(2, L[1, k])=\phi(3, k) \phi(2, L[1, k])=1$ for all $k \leq 4$.

- If $(i, j)=(2,2)$, then $\phi(1, k) \phi(2,2) \phi(2, k) \phi(2, L[2, k])=\phi(2, k) \phi(2, L[2, k])=1$ for all $k \leq 4$.

\section{Pseudocoboundaries and Pseudococycles over Latin Rectangles}

In this section, we introduce the notions of both the pseudocoboundary and pseudococycle over a Latin rectangle as a natural generalization of the similar concepts described over quasigroups in [17] by keeping in mind, to this end, the concepts introduced in [18]. Firstly, let us define the types of Latin rectangles where such a generalization is feasible.

Let $r$ and $n$ be two positive integers such that $r \leq n$, and let $L \in \mathcal{R}_{r, n}$ be such that

$$
L[L[i, j], k] \neq L[i, L[j, k]],
$$

for some triple $(i, j, k) \in[r] \times[r] \times[n]$ such that $L[i, j] \leq r$. We apply the term "nonassociative" to any such triple satisfying Condition (4). Let $N S(L)$ denote from here on the set of such non-associative triples within the Latin rectangle $L$. The cardinality of this set is the index of non-associativity of $L$, which is denoted by $n s(L)$. If $r=n$, then Condition (4) implies that the associative property does not hold for the triple $(i, j, k)$ in the non-associative quasigroup with $L$ as its Cayley table. In this case, the index $n s(L)$ measures the associativity of that quasigroup. This index has been studied for different types of algebraic structures [39-42] since it was introduced in 1947 by Climescu [43] for any given multiplicative system. Particularly, it is easily verified [44] that $n s(L) \leq n^{3}-n$ for every Latin square $L$ of order $n$. This upper bound has recently been proved [32] to be sharp for order $n>1$. Furthermore, it is also known [45] that $16 n-64 \leq n s(L)$ for every Latin square of even order $n \geq 168$. The reader is also referred to [46,47] for some other studies dealing with the number of non-associative triples of a Latin square.

In this paper, we are interested in the Latin rectangles $L \in \mathcal{R}_{r, n}$ such that $n s(L)>0$. The following lemma characterizes the case of $r=1$.

Lemma 1. Let $L \in \mathcal{R}_{1, n}$. Then, $n s(L)>0$ if and only if $L[1,1]=1$ and there exists a positive integer $k \leq n$ such that $L[1, k] \neq k$.

Proof. Notice from Condition (4) that every non-associative triple of the $1 \times n$ Latin rectangle $L$ would be of the form $(1,1, k)$ for some positive integer $k \leq n$ satisfying that $L[L[1,1], k] \neq L[1, L[1, k]]$. In addition, Condition (4) also implies that $L[1,1]=1$ and, hence, $L[1, k] \neq L[1, L[1, k]]$. As a consequence, $L[1, k] \neq k$.

Let $L \in \mathcal{R}_{r, n}$ be such that $n s(L)>0$. Every non-associative triple $(i, j, k) \in N S(L)$ is related to two distinct positive integers $h_{1}, h_{2} \leq n$ such that $h_{1}=L[L[i, j], k] \neq L[i, L[j, k]]=$ $h_{2}$. From here on, let $\mathcal{H}(L)$ denote the set of positive integers $h \leq n$ such that

$$
\{h\} \subset\{L[L[i, j], k], L[i, L[j, k]]\},
$$

for some $(i, j, k) \in N S(L)$. It is readily verified that $n s(L)=0$ whenever $n \leq 2$. So, from now on, we suppose that $n>2$ throughout the paper. Notice also that every Latin square in $\mathcal{R}_{n, n}$ with $n s(L)>0$ is the Cayley table of a non-associative quasigroup of order $n$. The case $r<n$ is illustrated by the following example. 
Example 2. Let us consider the Latin rectangle L that is described in Example 1. Then,

$$
N S(L)=\{(1,1,3),(1,1,4),(1,2,1),(1,2,4),(2,2,1),(2,2,2),(2,2,3),(2,2,4)\} .
$$

Hence, $n s(L)=8$. In addition, $\mathcal{H}(L)=[4]$. To prove it, take, for instance, the triples $(2,2,1)$ and $(1,2,1)$ in $N S(L)$.

Let $L \in \mathcal{R}_{r, n}$ be such that $n s(L)>0$ and let $h \in \mathcal{H}(L)$. We define the $h$-pseudocoboundary over the Latin rectangle $L$ as the map $\psi_{L ; h}:[r] \times[n] \rightarrow\{-1,1\}$, which is described so that

$$
\psi_{L, h}(i, j):=\partial_{h}(i) \partial_{h}(j) \partial_{h}(L[i, j]),
$$

for all positive integers $i \leq r$ and $j \leq n$, where

$$
\partial_{h}(k):=\left\{\begin{aligned}
-1, & \text { if } k=h \\
1, & \text { otherwise. }
\end{aligned}\right.
$$

In addition, we apply the term " $h$-pseudocoboundary matrix" over $L$ to the $r \times n$ matrix $M_{\psi_{L ; h}}:=\left(\psi_{L ; h}(i, j)\right)_{(i, j) \in[r] \times[n]}$. When we want to refer to any $h$-pseudocoboundary (matrix) over $L$, we omit the prefix $h$. As such, the concept of the pseudocoboundary over a Latin rectangle constitutes a generalization of that over a quasigroup [17], which arises when $r=n$. In any case, the following result establishes that the pseudococyclic framework over Latin rectangles is not included in the cocyclic framework over such arrays. Hence, it constitutes a new proposal that has to be independently studied.

Lemma 2. Let $L \in \mathcal{R}_{r, n}$ be such that $n s(L)>0$ and let $h \in \mathcal{H}(L)$. The h-pseudococycle $\psi_{L ; h}$ is not a cocycle over $L$.

Proof. Let us see that the $h$-pseudocycle $\psi_{L ; h}$ does not hold the cocycle Equation (3). To this end, let $(i, j, k) \in N S(L)$ be such that Condition (5) holds. Then,

$$
\psi_{L ; h}(L[i, j], k) \psi_{L ; h}(i, j) \psi_{L ; h}(j, k) \psi_{L ; h}(i, L[j, k])=\partial_{h}(L[L[i, j], k]) \partial_{h}(L[i, L[j, k]])=-1 .
$$

Let us illustrate all of these concepts with a series of examples.

Example 3. Let $L$ be the Latin rectangle described in Example 1. According to Example 2, we can define four pseudocoboundaries over $L$, which are represented by the following matrices.

$$
\begin{aligned}
& M_{\psi_{L ; 1}} \equiv \begin{array}{|c|c|c|c|}
\hline- & - & - & - \\
\hline- & - & + & + \\
\hline
\end{array} \\
& M_{\psi_{L ; 2}} \equiv \begin{array}{|c|c|c|c|}
\hline+ & + & + & + \\
\hline- & + & + & - \\
\hline
\end{array} \\
& M_{\psi_{L ; 3}} \equiv \begin{array}{|c|c|c|c|}
\hline+ & + & - & - \\
\hline- & + & - & + \\
\hline
\end{array} \\
& M_{\psi_{L ; 4}} \equiv \begin{array}{|c|c|c|c|}
\hline+ & + & - & - \\
\hline+ & + & + & + \\
\hline
\end{array}
\end{aligned}
$$

The following example enables us to ensure that, unlike the cocyclic development over quasigroups, there exist Hadamard matrices that are pseudocoboundary matrices over quasigroups that are not loops. 
Example 4. Let us consider the Latin square

$$
L \equiv \begin{array}{|l|l|l|l|}
\hline 1 & 2 & 4 & 3 \\
\hline 2 & 1 & 3 & 4 \\
\hline 3 & 4 & 1 & 2 \\
\hline 4 & 3 & 2 & 1 \\
\hline
\end{array}
$$

We have that $n s(L)=32$ and $\mathcal{H}(L)=[4]$. In order to prove this last end, take, for instance, the subset $\{(1,1,3),(1,3,3)\} \subset N S(L)$. It is simply verified that every h-pseudocoboundary matrix of $L$ with $1 \leq h \leq 4$ is Hadamard.

$M_{\psi_{L ; 1}} \equiv$\begin{tabular}{|c|c|c|c|}
\hline- & - & - & - \\
\hline- & - & + & + \\
\hline- & + & - & + \\
\hline- & + & + & -
\end{tabular}$\quad M_{\psi_{L ; 2}} \equiv$\begin{tabular}{c|c|c|c|c|}
+ & + & + & + \\
\hline+ & + & - & - \\
\hline+ & - & + & - \\
\hline+ & - & - & + \\
\hline
\end{tabular}$\quad M_{\psi_{L ; 3}}=M_{\psi_{L ; 4}} \equiv \quad$\begin{tabular}{c|c|c|c|c|}
\hline+ & + & - & - \\
\hline+ & + & + & + \\
\hline+ & - & + & - \\
\hline+ & - & - & + \\
\hline
\end{tabular}

Observe that all the pseudocoboundary matrices shown in Examples 3 and 4 constitute (partial) Hadamard matrices. Proposition 2 described in Section 4 enables us to ensure that this condition does not hold in general. Finally, the following example enables us to ensure the existence of Hadamard matrices that are not cocyclic over any Latin rectangle, but that are pseudocoboundary matrices over a Latin square.

Example 5. It is known ([18] Example 41) that the following Hadamard matrix is not cocyclic over any Latin rectangle.

$$
M \equiv\left(\begin{array}{cccc}
+ & + & + & + \\
- & + & + & - \\
- & - & + & + \\
+ & - & + & -
\end{array}\right)
$$

Nevertheless, it constitutes a 2-pseudocoboundary matrix over the Latin square

$$
L \equiv \begin{array}{|l|l|l|l|}
\hline 1 & 2 & 3 & 4 \\
\hline 3 & 4 & 2 & 1 \\
\hline 2 & 1 & 4 & 3 \\
\hline 4 & 3 & 1 & 2 \\
\hline
\end{array}
$$

Let us finish this section by introducing the notion of a pseudococycle over a Latin rectangle as a generalization of both the concepts of a cocycle over a Latin rectangle [18] and a pseudococycle over a quasigroup [17]. To this end, we take into account the previously described notion of a pseudocoboundary over Latin rectangles. Thus, we define a pseudococycle over a given Latin rectangle $L \in \mathcal{R}_{r, n}$ with $n s(L)>0$ as any map

$$
\psi=\left(\prod_{h \in S \subseteq \mathcal{H}(L)} \psi_{h}\right) \phi
$$

that is obtained as the product of some $h$-pseudocoboundaries with $h \in S \subseteq \mathcal{H}(L)$ and a cocycle $\phi$, all of them over the Latin rectangle $L$. It is represented by the pseudococyclic matrix $M_{\psi}:=(\psi(i, j))_{(i, j) \in[r] \times[n]}$. In particular, notice from this definition that every pseudocoboundary over a Latin rectangle is a pseudococycle over the latter by means of the trivial cocycle. Further, if $S=\varnothing$, then all of these concepts refer to the cocyclic framework over Latin rectangles, whose fundamentals were comprehensively studied in [18].

In a similar way, if $r=n$, then they refer to the pseudococyclic framework over quasigroups, which has only been briefly dealt with in [17]. This paper focuses, therefore, on the fundamentals of the case $S \neq \varnothing$, whatever the positive integer $r \leq n$ is. The following example illustrates this case. 
Example 6. Let $L$ be the Latin rectangle described in Example 1. Then, the following assertions are readily verified from the cocyclic matrix $M_{\phi}$ described in that example, together with the pseudocoboundary matrices $M_{\psi_{L ; 3}}$ and $M_{\psi_{L ; 4}}$ described in Example 3.

- $\quad$ The pseudococyclic matrix over $L$ that is associated with the pseudococycle $\psi_{L ; 3} \psi_{L ; 4}$ is partial Hadamard.

$$
M_{\psi_{L ; 3} \psi_{L ; 4}} \equiv \begin{array}{|c|c|c|c|}
\hline+ & + & + & + \\
\hline- & + & - & + \\
\hline
\end{array}
$$

- $\quad$ The pseudococyclic matrix over $L$ that is associated with the pseudococycle $\psi_{L ; 3} \psi_{L ; 4} \phi$ is not a pseudococyclic partial Hadamard matrix.

$$
M_{\psi_{L ; 3} \psi_{L ; 4} \phi} \equiv \begin{array}{|c|c|c|c|}
\hline+ & + & + & + \\
\hline- & + & - & - \\
\hline
\end{array}
$$

\section{Pseudocoboundary Partial Hadamard Matrices over Latin Rectangles}

Let us start our study by dealing with Problem 1 concerning the conditions under which we can ensure the existence of pseudocoboundary partial Hadamard matrices over a given Latin rectangle $L \in \mathcal{R}_{r, n}$ with $n s(L)>0$. Firstly, we focus on the case $r=1$.

Proposition 1. There always exists a pseudocoboundary partial Hadamard matrix over a Latin rectangle $L \in \mathcal{R}_{1, n}$ satisfying that $n s(L)>0$.

Proof. Let $L \in \mathcal{R}_{1, n}$ be such that $n s(L)>0$. From Lemma 1 , it must be $L[1,1]=1$ and $L[1, j]=h$ for some positive integers $j, h \leq n$ such that $1 \neq j \neq h \neq 1$. Hence, the Latin rectangle condition of no repetition of symbols in each row implies that $L[L[1,1], j]=h \neq$ $L[1, h]=L[1, L[1, j]]$. Thus, $(1,1, j) \in N S(L)$ and $h \in \mathcal{H}(L)$. The matrix $\psi_{L ; h}$ is trivially partial Hadamard over $L$.

Let us focus now on the case $r>1$. Since $n s(L)=0$ for all $L \in \mathcal{R}_{2,2}$, we also suppose that the number $n \geq r$ of columns is a multiple of 4 . We start with a preliminary lemma that describes the entries within each row and column of any pseudocoboundary partial Hadamard matrix over a given Latin rectangle. Particularly, it characterizes the rows and columns that are uniformly signed.

Lemma 3. Let $r$ and $n$ be two positive integers such that $2 \leq r \leq n$. Further, let $\psi_{L ; h}$ be the h-pseudocoboundary over a Latin rectangle $L \in \mathcal{R}_{r, n}$ with $n s(L)>0$ and $h \in \mathcal{H}(L)$. Then, the following assertions hold.

1. Let $i \leq r$ be such that $L[i, h] \neq h$. Then,

$$
\psi_{L ; h}(i, j)=\left\{\begin{aligned}
-\partial_{h}(i), & \text { if either } j=h \text { or } L[i, j]=h \\
\partial_{h}(i), & \text { otherwise. }
\end{aligned}\right.
$$

2. Let $j \leq n$. Then,

$$
\psi_{L ; h}(i, j)=\left\{\begin{aligned}
-\partial_{h}(j), & \text { if }\left\{\begin{array}{l}
h \leq r, L[h, j] \neq h \text { and either } i=h \text { or } L[i, j]=h, \\
h>r \text { and } L[i, j]=h,
\end{array}\right. \\
\partial_{h}(j), & \text { otherwise. }
\end{aligned}\right.
$$

3. The ith row of the h-pseudocoboundary matrix $M_{\psi_{L ; h}}$ with $i \leq r$ is uniformly signed if and only if $L[i, h]=h$. In such a case, $\psi_{L ; h}(i, j)=\partial_{h}(i)$ for all $j \leq n$. As a consequence, there always exists at most one uniformly signed row.

4. Let $j \leq n$. If $h>r$, then the $j$ th column of $M_{\psi_{h}}$ is uniformly signed if and only if $L[i, j] \neq h$ for every positive integer $i \leq r$. Otherwise, if $h \leq r$, then the jth column of $M_{\psi_{h}}$ is uniformly signed if $L[h, j]=h$. If $r>2$, then this sufficient condition is also necessary. In any case, 
$\psi_{L ; h}(i, j)=\partial_{h}(j)$ for all $i \leq r$. Furthermore, there exists exactly one uniformly signed column if $h \leq r$ and $r>2$.

Proof. The first two assertions and the sufficient conditions of the last two assertions follow from the Definition (6). Let us focus now on the proof of the necessary condition of the third assertion (that one of the four statements follows similarly). Thus, let us suppose the existence of a positive integer $i \leq r$ such that the $i$ th row of the $h$-pseudocoboundary matrix $M_{\psi_{L ; h}}$ is uniformly signed. Then, the mentioned Definition (6) implies that either $\partial_{h}(L[i, j])=\partial_{h}(j)$ or $\partial_{h}(L[i, j])=-\partial_{h}(j)$ for all $j \leq n$. Nevertheless, since $n>2$, the definition of the map $\partial_{h}$, together with the Latin rectangle condition of no repetitions of symbols per row, implies that the second option is not possible. Hence, it must be $L[i, h]=h$. The final consequence described in the third assertion holds straightforwardly from the Latin rectangle condition of no repetitions of symbols in each column.

Concerning the last sentence of the fourth assertion, the definition of the map $\partial_{h}$, together with (6) and the Latin rectangle condition of no repetitions of symbols per row, implies the existence of exactly one uniformly signed column when $r>2$.

Example 7. Let $L$ be the Latin rectangle described in Example 1. The third assertion of Lemma 3 explains, for instance, the uniformity of signs of the first row of both matrices $M_{\psi_{L ; 1}}$ and $M_{\psi_{L ; 2}}$, and also of the second row of the matrix $M_{\psi_{L ; 4}}$, all of them described in Example 3. In addition, it also explains that there does not exist any uniformly signed row in the matrix $M_{\psi_{L ; 3}}$.

The fourth assertion of Lemma 3 explains, for instance, the uniformity of signs of the first column of $M_{\psi_{L ; 1}}$ and the third column of $M_{\psi_{L ; 2}}$. It also explains the two uniformly signed columns of both matrices $M_{\psi_{L ; 3}}$ and $M_{\psi_{L ; 4}}$. Nevertheless, this fourth assertion of Lemma 3 does not explain the uniformity of signs of the second columns of $M_{\psi_{L ; 1}}$ and $M_{\psi_{L ; 2}}$, which follows indeed from the second assertion of this lemma. It illustrates, in particular, the exceptional case $r=2$ that was discarded therein. The case $r>2$ is illustrated by the existence of exactly one uniformly signed column in any of the Latin squares described in Examples 4 and 5.

The following result characterizes the Latin rectangles over which a pseudocoboundary partial Hadamard matrix exists. As such, it constitutes, together with Proposition 1, the answer to Problem 1.

Proposition 2. Let $r$ and $n$ be two positive integers such that $2 \leq r \leq n$. Further, let $\psi_{L ; h}$ be the $h$-pseudocoboundary over a Latin rectangle $L \in \mathcal{R}_{r, n}$ with $n s(L)>0$ and $h \in \mathcal{H}(L)$. Then, the pseudocoboundary matrix $M_{\psi_{L ; h}}$ is partial Hadamard if and only if $n=4$.

Proof. Lemma 3 enables us to ensure that the $h$-pseudocoboundary $\psi_{L ; h}$ has at least $r-2$ rows with precisely two negative entries. Hence, the pseudocoboundary matrix $M_{\psi_{L ; h}}$ cannot be Hadamard if $n>4$. Concerning the case $n=4$, let us remind the reader that there exist 576 Latin squares of order four, from which only 16 of them constitute the Cayley table of an associative quasigroup. A simple and exhaustive computation enables us to ensure that $\mathcal{H}(L)=[4]$ for all of the 560 remaining Latin squares $L \in \mathcal{R}_{4,4}$, and also that all of their related $h$-pseudocoboundary matrices are partial Hadamard, whatever the positive integer $h \leq 4$ is. As a consequence, every $h$-pseudocoboundary matrix of an $r \times 4$ Latin rectangle is partial Hadamard, whatever the two positive integers $h, r \leq 4$ are.

For Latin squares of any given order, the following result holds as an immediate consequence of Lemma 3, once it is noticed that its two last assertions always hold in the case of $L$ being a Latin square. It is illustrated by any of the pseudocoboundary matrices described in Examples 4 and 5.

Proposition 3. Let $\psi_{L ; h}$ be the h-pseudocoboundary over a Latin square of order $n>2$ with $n s(L)>$ 0 and $h \in \mathcal{H}(L)$. Then, the h-pseudocoboundary matrix $M_{\psi_{L ; h}}$ contains exactly one uniformly signed row and exactly one uniformly signed column. 
Let us finish this section by focusing on Problem 2 concerning the conditions under which a given partial Hadamard matrix is a pseudocoboundary over some Latin rectangle $L \in \mathcal{R}_{r, n}$ with $n s(L)>0$. From Proposition 2, we may assume $n=4$. Firstly, we focus on the case $r=1$. Notice in this regard that every $1 \times n$ binary array trivially constitutes a partial Hadamard matrix by itself.

Lemma 4. Let $M=\left(m_{1 j}\right)$ be a $1 \times 4$ partial Hadamard matrix. It is a pseudocoboundary matrix over a Latin rectangle if and only if $m_{11}=1$ and it contains exactly two negative entries.

Proof. In order to prove the necessary condition, let us suppose that the partial Hadamard matrix $M$ is an $h$-pseudocoboundary over a Latin rectangle $L \in \mathcal{R}_{1,4}$ with $h \in \mathcal{H}(L) \neq \varnothing$. From Lemma 1, it must be $L[1,1]=1$, and then, the Latin rectangle condition of no repetitions of symbols in each row implies that $(1,1,1) \notin N S(L)$ and $1 \notin \mathcal{H}(L)$. Hence, $h \neq 1$ and $m_{11}=1$. In addition, since every non-associative triple in $N S(L)$ is of the form $(1,1, k)$ with $k \in\{2,3,4\}$ and $h \in \mathcal{H}(L)$, it should be $\{h\} \subset\left\{L\left[L[1,1], k_{0}\right], L\left[1, L\left[1, k_{0}\right]\right]\right\}=$ $\left\{L\left[1, k_{0}\right], L\left[1, L\left[1, k_{0}\right]\right]\right\}$ for some positive integer $k_{0} \in\{2,3,4\}$. If $k_{0}=h$, then we get $\{h\} \subset\{h\}$, which is a contradiction. So, $L[1, h] \neq h$, and hence, the matrix $M$ contains exactly two negative entries. More specifically, $m_{1 k_{0}}=m_{1 h}=-1$.

Now, in order to prove the sufficient condition, let us suppose that $m_{11}=1$ and let $h, i, j \in[4] \backslash\{1\}$ be three distinct positive integers such that $m_{1, h}=m_{1, i}=-1$ and $m_{1, j}=1$. Then, let $L \in \mathcal{R}_{1,4}$ be defined so that $L[1,1]=1, L[1, h]=j, L[1, i]=h$, and $L[1, j]=i$. Then, $L[L[1,1], h]=j \neq i=L[1, L[1, h]]$. Hence, $(1,1, h) \in N S(L)$ and $h \in \mathcal{H}(L)$. It is straightforwardly verified that the partial Hadamard matrix $M$ is an $h$-pseudocoboundary over $L$.

Let us focus now on the case $2 \leq r \leq 4$. The following preliminary lemma holds straightforwardly from the definition (6) of a pseudocoboundary.

Lemma 5. Let $r \in\{2,3,4\}$ and let $M=\left(m_{i j}\right)$ be an $r \times 4$ partial Hadamard matrix such that there exists a Latin rectangle $L \in \mathcal{R}_{r, 4}$ with $n s(L)>0$, over which $M$ is an h-pseudocoboundary matrix for some $h \in \mathcal{H}(L)$. The following assertions hold.

1. The ith row of the partial Hadamard matrix $M$ with $i \leq r$ is uniformly signed if and only if $L[i, h]=h$. In such a case, $m_{i j}=\partial_{h}(i)$ for all $j \leq 4$.

2. If $h \leq r$ and $L[h, h]=h$, then $m_{i h}=-1$ for all $i \leq r$. Moreover, if $m_{i j}=-1$ with $i \neq h \neq j$ then $L[i, j]=h$.

3. If $L[i, h]=h$ for some $i \in[r] \backslash\{h\}$, then $m_{j h}=-\partial_{h}(j)$ for all $j \in[r] \backslash\{i\}$. Moreover, if $m_{j k}=-\partial_{h}(j)$ for some $j \in[r] \backslash\{i\}$ and $k \in[4] \backslash\{h\}$, then $L[j, k]=h$.

Example 8. Let us consider the following four partial Hadamard matrices.

$$
\begin{aligned}
& M_{1} \equiv \begin{array}{|c|c|c|c|}
\hline- & - & - & - \\
\hline+ & - & - & + \\
\hline
\end{array} \\
& M_{3} \equiv \begin{array}{|c|c|c|c|}
\hline+ & + & + & + \\
\hline+ & + & - & - \\
\hline
\end{array}
\end{aligned}
$$$$
M_{2} \equiv \begin{array}{|l|l|l|l|}
\hline- & - & - & - \\
\hline- & - & + & + \\
\hline
\end{array}
$$$$
M_{4} \equiv \begin{array}{|c|c|c|c|}
\hline+ & + & + & + \\
\hline- & + & - & + \\
\hline
\end{array}
$$

Let $N=\left(n_{i j}\right) \in\left\{M_{1}, M_{2}\right\}$. The first statement of Lemma 5 enables us to ensure that, if the matrix $N$ were an h-pseudocoboundary over some Latin rectangle $L \in \mathcal{R}_{2,4}$ with $h \in \mathcal{H}(L) \neq \varnothing$, then it should be $h=1$ and $L[1,1]=1$. However, then, the second statement of the mentioned lemma implies that $n_{2,1}=-1$, which is not the case when $N=M_{1}$. As a consequence, the partial Hadamard matrix $M_{1}$ is not a pseudocoboundary over any Latin rectangle. Further, concerning the case $N=M_{2}$, the second statement of Lemma 5 also enables us to ensure that $L[2,2]=1$. Thus, for instance, it is readily verified that the matrix $M_{2}$ is a 1-pseudocoboundary over the Latin rectangle 


$$
L \equiv \begin{array}{|l|l|l|l|}
\hline 1 & 4 & 2 & 3 \\
\hline 2 & 1 & 3 & 4 \\
\hline
\end{array}
$$

In particular, $(2,1,3) \in N S(L)=\{(1,1,2),(1,1,3),(1,1,4),(2,1,2),(2,1,3),(2,1,4)$, $(2,2,2),(2,2,3),(2,2,4)\}$ and $1 \in \mathcal{H}(L)=[4]$. More specifically, $L[L[2,1], 3]=3 \neq 1=$ $L[2, L[1,3]]$.

Notice also that the partial Hadamard matrices $M_{3}$ and $M_{4}$ are, respectively, 2-pseudocoboundaries over the Latin rectangles

$$
L^{\prime} \equiv \begin{array}{|l|l|l|l|}
\hline 3 & 2 & 1 & 4 \\
\hline 2 & 4 & 3 & 1
\end{array} \quad \text { and } \quad L^{\prime \prime} \equiv \begin{array}{|l|l|l|l|}
\hline 1 & 2 & 3 & 4 \\
\hline 3 & 1 & 4 & 2 \\
\hline
\end{array}
$$

In particular, $(2,1,3) \in N S\left(L^{\prime}\right)=\{(1,2,3),(1,2,4),(2,1,1),(2,1,3)\}$ and $2 \in \mathcal{H}\left(L^{\prime}\right)=$ $\{1,2,3\}$. More specifically, $L^{\prime}\left[L^{\prime}[2,1], 3\right]=3 \neq 2=L^{\prime}\left[2, L^{\prime}[1,3]\right]$. Further, concerning the Latin rectangle $L^{\prime \prime}$, we have that $(2,2,2) \in N S\left(L^{\prime \prime}\right)=\{(2,2,1),(2,2,2),(2,2,3),(2,2,4)\}$ and $2 \in \mathcal{H}\left(L^{\prime \prime}\right)=[4]$. In fact, $L^{\prime \prime}\left[L^{\prime \prime}[2,2], 2\right]=2 \neq 3=L^{\prime \prime}\left[2, L^{\prime \prime}[2,2]\right]$.

The following result characterizes the partial Hadamard matrices that have a uniformly signed row with all its entries being negative, which are pseudocoboundaries over a Latin rectangle. Its constructive proof is illustrated by the matrix $M_{2}$ and the Latin rectangle $L$ described in Example 8.

Proposition 4. Let $M=\left(m_{i j}\right)$ be an $r \times 4$ partial Hadamard matrix with $r>1$ such that $m_{h j}=-1$ for some $h \leq r$ and all $j \leq 4$. It is a pseudocoboundary matrix over a Latin rectangle if and only if $m_{\text {ih }}=-1$ for all $i \leq r$.

Proof. The necessary condition follows from Lemma 5. Now, in order to prove the sufficient condition, let $i \in[r] \backslash\{h\}$ and $j \in[4] \backslash\{h\}$ be such that $m_{i j}=-1$. It always exists because $M$ is an $r \times 4$ partial Hadamard matrix with $r>1$. In addition, let $k \in[4] \backslash\{h\}$ be such that $i \neq k \neq j$. Finally, let $L$ be any $r \times 4$ Latin rectangle satisfying that $L[h, h]=L[i, j]=h$, $L[h, k]=j, L[i, h]=i$, and $L[i, k]=k$. Moreover, it must be $L\left[i^{\prime}, j^{\prime}\right]=h$ for all $i^{\prime} \in[r] \backslash\{h\}$ and $j^{\prime} \in[4] \backslash\{h\}$ such that $m_{i^{\prime} j^{\prime}}=-1$. In particular, $L[L[i, h], k]=k \neq h=L[i, L[h, k]]$. Hence, $(i, h, k) \in N S(L)$ and $h \in \mathcal{H}(L)$. It is simply verified that the partial Hadamard matrix $M$ is $h$-pseudocoboundary over $L$.

In a similar way, the next result characterizes the partial Hadamard matrices have a uniformly signed row with all its entries being positive, which are pseudocoboundaries over a Latin rectangle. The two subcases described in its constructive proof are respectively illustrated by the matrices $M_{3}$ and $M_{4}$, together with the Latin rectangles $L^{\prime}$ and $L^{\prime \prime}$, which are described in Example 8.

Proposition 5. Let $M=\left(m_{i j}\right)$ be an $r \times 4$ partial Hadamard matrix with $r>1$ such that $m_{i j}=1$ for some positive integer $i \leq r$ and all $j \leq 4$. It is a pseudocoboundary matrix over a Latin rectangle if and only if there exists a positive integer $h \in[r] \backslash[i]$ such that $m_{j h}=-\partial_{h}(j)$ for all $j \in[r] \backslash\{i\}$.

Proof. Again, the necessary condition follows from Lemma 5. Now, in order to prove the sufficient condition, let $j \in[4] \backslash\{h\}$ be such that $m_{h j}=1$. It always exists because $M$ is an $r \times 4$ partial Hadamard matrix with $r>1$. The following two cases arise.

- If $j=i$, then let $k \in[4] \backslash\{h, i\}$ and let $L$ be any $r \times 4$ Latin rectangle satisfying that $L[i, h]=L[h, i]=h, L[i, k]=i$ and $L[h, k]=k$. In addition, it must be $L\left[i^{\prime}, j^{\prime}\right]=h$ for all $i^{\prime} \in[r] \backslash\{i\}$ and $j^{\prime} \in[4] \backslash\{h\}$ such that $m_{i^{\prime} j^{\prime}}=-\partial_{h}\left(i^{\prime}\right)$. Then, $L[L[h, i], k]=k \neq$ $h=L[h, L[i, k]]$. 
- If $j \neq i$, then let $L$ be any $r \times 4$ Latin rectangle satisfying that $L[i, h]=L[h, j]=h$, $L[h, h]=i$, and $L[h, i]=j$. Again, we also impose that $L\left[i^{\prime}, j^{\prime}\right]=h$ for all $i^{\prime} \in[r] \backslash\{i\}$ and $j^{\prime} \in[4] \backslash\{h\}$ such that $m_{i^{\prime} j^{\prime}}=-\partial_{h}\left(i^{\prime}\right)$. Then, $L[L[h, h], h]=h \neq j=L[h, L[h, h]]$.

In any case, $h \in \mathcal{H}(L)$, and thus, the partial Hadamard matrix $M$ is an $h$-pseudocoboundary over $L$.

Finally, in order to give a complete answer to Problem 2, the following result characterizes the $r \times 4$ partial Hadamard matrices with $r>1$ and without uniformly signed rows, which are pseudocoboundaries over a Latin rectangle. Example 9 illustrates its constructive proof.

Proposition 6. Let $M=\left(m_{i j}\right)$ be an $r \times 4$ partial Hadamard matrix with $r>1$ and without uniformly signed rows. Then, the following assertions hold.

1. If $r=4$, then the matrix $M$ is not a pseudocoboundary over any Latin square of order four.

2. If $r \in\{2,3\}$, then the matrix $M$ is pseudocoboundary over an $r \times 4$ Latin rectangle if and only if the following two conditions hold.

(a) There exists a positive integer $h \leq 4$ such that $m_{i h}=-\partial_{h}(i)$ for all $i \leq r$.

(b) For each positive integer $i \leq r$, there exists exactly one positive integer $j_{i} \in[4] \backslash\{h\}$ such that $m_{i, j_{i}}=m_{i, h}$. Moreover, the set $\left\{j_{1}, \ldots, j_{r}\right\}$ is formed by $r$ distinct positive integers.

If this is the case, then the matrix $M$ is indeed an h-pseudocoboundary over an $r \times 4$ Latin rectangle.

Proof. Let us suppose that the partial Hadamard matrix $M$ is an $h$-pseudocoboundary over a Latin rectangle $L \in \mathcal{R}_{r, 4}$ with $n s(L)>0$ and $h \in \mathcal{H}(L)$. From the first assertion of Lemma 5, the non-existence of uniformly signed rows within $M$ implies that $L[i, h] \neq h$ for every positive integer $i \leq r$. It constitutes a contradiction when $r=4$ because of the Latin rectangle condition of no repetitions of symbols per column. Hence, the first assertion holds. Further, if $r \in\{2,3\}$, then $m_{i h}=\psi_{L ; h}(i, h)=\partial_{h}(i) \partial_{h}(h) \partial_{h}(L[i, h])=-\partial_{h}(i)$ for every positive integer $i \leq r$. Similarly, it is readily proven that the elements $j_{i}$ described in Condition (2b) refer to the columns in which the symbol $h$ appears in the $i$ th row of $L$. That is, $L\left[i, j_{i}\right]=h$ for all $i \leq r$. Notice that all these columns are pairwise distinct from the Latin rectangle condition of no repetition of symbols in each column.

In order to prove the sufficient condition of the second assertion, let us suppose that both Conditions (2a) and (2b) hold. Then, let $L$ be any $r \times 4$ Latin rectangle satisfying that $L[i, h] \neq h=L\left[i, j_{i}\right]$ for every positive integer $i \leq r$. The following two cases arise.

- If $h \leq r$, then let us consider a positive integer $i \in[r] \backslash\{h\}$. It exists because $r \geq 2$. The following two subcases arise.

- If $i=j_{i}$, then let us impose that $L\left[i, j_{h}\right]=j_{h}$. Then, $L\left[L[i, i], j_{h}\right]=h \neq j_{h}=$ $L\left[i, L\left[i, j_{h}\right]\right]$.

- $\quad$ If $i \neq j_{i}$, then let us impose that $L[i, h]=i, L\left[i, j_{h}\right]=j_{i}$ and $L\left[h, j_{i}\right]=j_{h}$. Under such assumptions, we have that $L\left[L[i, h], j_{i}\right]=h \neq j_{i}=L\left[i, L\left[h, j_{i}\right]\right]$.

- If $h>r$, then let us consider a pair of distinct positive integers $i_{1}, i_{2} \leq r$. Notice again to this end that $r \geq 2$. Similarly to the previous case, the following two subcases arise.

- Firstly, let us suppose the existence of a positive integer $i \in\left\{i_{1}, i_{2}\right\}$ such that $i=j_{i}$. Without loss of generality, we can suppose that $i_{1}=j_{i_{1}}$. Then, let us impose that $L\left[i_{2}, i_{1}\right]=i_{1}$ and $L\left[i_{2}, h\right]=i_{2}$. Under such assumptions, we have that $L\left[L\left[i_{2}, i_{1}\right], i_{1}\right]=h \neq i_{2}=L\left[i_{2}, L\left[i_{1}, i_{1}\right]\right]$.

- Otherwise, let us suppose that $i_{1} \neq j_{i_{1}}$ and $i_{2} \neq j_{i_{2}}$. Then, let us impose that $L\left[i_{1}, i_{1}\right]=i_{1}$ and $L\left[i_{1}, h\right]=i_{2}$. Under such assumptions, we have that $L\left[L\left[i_{1}, i_{1}\right], j_{i_{1}}\right]=h \neq i_{2}=L\left[i_{1}, L\left[i_{1}, j_{i_{1}}\right]\right]$.

In any case, $h \in \mathcal{H}(L)$, and thus, the partial Hadamard matrix $M$ is an $h$-pseudocoboundary over $L$. 
Example 9. Let us consider the following six partial Hadamard matrices.

$$
\begin{aligned}
& M_{1} \equiv \begin{array}{|c|c|c|c|}
\hline+ & - & + & + \\
\hline+ & - & - & - \\
\hline+ & + & + & - \\
\hline
\end{array} \\
& M_{3} \equiv \begin{array}{|c|c|c|c|}
\hline- & - & + & + \\
\hline- & + & + & - \\
\hline+ & - & + & - \\
\hline
\end{array} \\
& M_{5} \equiv \begin{array}{|c|c|c|c|}
\hline- & + & + & - \\
\hline+ & + & - & - \\
\hline+ & - & + & - \\
\hline
\end{array}
\end{aligned}
$$$$
M_{2} \equiv \begin{array}{|c|c|c|c|}
\hline+ & - & + & + \\
\hline+ & + & + & - \\
\hline+ & - & - & - \\
\hline
\end{array}
$$$$
M_{4} \equiv \begin{array}{|c|c|c|c|}
\hline+ & - & - & + \\
\hline- & + & - & + \\
\hline- & - & + & + \\
\hline
\end{array}
$$

$$
M_{6} \equiv \begin{array}{|c|c|c|c|}
\hline+ & + & - & - \\
\hline- & + & + & - \\
\hline+ & - & + & - \\
\hline
\end{array}
$$

Condition (2a) in Proposition 6 implies that $M_{1}$ is not a pseudocoboundary over any $3 \times 4$ Latin rectangle. It also enables us to ensure that the only possibility to get $M_{2}$ to be an h-pseudocoboundary over some $3 \times 4$ Latin rectangle for some positive integer $h \leq 4$ is by considering $h=2$. However, then, Condition (2b) implies that it neither is an option because, for instance, the first row only contains one negative sign.

On the other hand, the partial Hadamard matrix $M_{3}$ is a 2-pseudocoboundary over the Latin rectangle

$$
L \equiv \begin{array}{|l|l|l|l|}
\hline 2 & 1 & 3 & 4 \\
\hline 1 & 4 & 2 & 3 \\
\hline 4 & 3 & 1 & 2 \\
\hline
\end{array}
$$

Here, $n s(L)=20,(1,1,3) \in N S(L)$, and $2 \subset \mathcal{H}(L)=[4]$. More specifically, $L[L[1,1], 3]=$ $2 \neq 3=L[1, L[1,3]]$.

Further, the partial Hadamard matrices $M_{4}$ and $M_{5}$ are, respectively, 2- and 4-pseudocoboundaries over the Latin rectangle

$$
L^{\prime} \equiv \begin{array}{|l|l|l|l|}
\hline 4 & 1 & 2 & 3 \\
\hline 1 & 3 & 4 & 2 \\
\hline 2 & 4 & 3 & 1 \\
\hline
\end{array} .
$$

In particular, $n s\left(L^{\prime}\right)=16,\{(1,2,3),(2,1,1)\} \subset N S\left(L^{\prime}\right)$, and $\{2,4\} \subset \mathcal{H}\left(L^{\prime}\right)=[4]$. More specifically, $L^{\prime}\left[L^{\prime}[1,2], 3\right]=2 \neq 3=L^{\prime}\left[1, L^{\prime}[2,3]\right]$ and $L^{\prime}\left[L^{\prime}[2,1], 1\right]=4 \neq 2=$ $L^{\prime}\left[2, L^{\prime}[1,1]\right]$.

Finally, the partial Hadamard matrix $M_{6}$ is a 4-pseudocoboundary over the Latin rectangle

$$
L^{\prime \prime} \equiv \begin{array}{|l|l|l|l|}
\hline 1 & 3 & 4 & 2 \\
\hline 4 & 1 & 2 & 3 \\
\hline 2 & 4 & 3 & 1 \\
\hline
\end{array} \text {. }
$$

Particularly, $n s\left(L^{\prime \prime}\right)=18,(1,1,3) \in N S(L)$, and $4 \in \mathcal{H}\left(L^{\prime \prime}\right)=[4]$. More specifically, $L^{\prime \prime}\left[L^{\prime \prime}[1,1], 3\right]=4 \neq 2=L^{\prime \prime}\left[1, L^{\prime \prime}[1,3]\right]$.

\section{Pseudococyclic Partial Hadamard Matrices Associated with the Trivial Cocycle}

Let us focus now on the characterization of the Latin rectangles $L \in \mathcal{R}_{r, n}$ with $n s(L)>0$ over which there exists a pseudococyclic partial Hadamard matrix. As a first stage, we focus in this section on the pseudococycles associated with the trivial cocycle; that is, on the pseudococycles of the form $\prod_{h \in S \subset \mathcal{H}(L)} \psi_{L ; h}$. Of course, the case $|S|=1$ corresponds to the pseudocoboundary framework that has already been studied in the previous subsection. It is so that we start with a generalization of Lemma 3 that describes the rows and columns of the pseudococyclic matrix associated with one such pseudococycle. To this end, for each 
given subset $S \subseteq \mathcal{H}(L)$ and each pair of positive integers $i \leq r$ and $j \leq n$, we previously define the sets

$$
\mathcal{D}_{L}^{-}(S, i):=\{k \in[n]: L[i, k] \in S\} \quad \text { and } \quad \mathcal{D}_{L}^{+}(S, j):=\{k \in[r]: L[k, j] \in S\} .
$$

If $r=n$, then the sets $\mathcal{D}_{L}^{-}(S, i)$ and $\mathcal{D}_{L}^{+}(S, i)$ constitute, respectively, the left division of $S$ by $i$ and the right division of $S$ by $j$, both of them within the quasigroup with the Latin square $L$ as its Cayley table. In addition, for all $r \leq n$, the Latin rectangle condition of no repetitions of symbols per row implies that $\left|\mathcal{D}_{L}^{-}(S, i)\right|=|S|$. Further, let $A \Delta B$ denote from here on the symmetric difference between two given sets $A$ and $B$.

Lemma 6. Let $\psi=\prod_{h \in S \subset \mathcal{H}(L)} \psi_{L ; h}$ be a pseudococycle over a Latin rectangle $L \in \mathcal{R}_{r, n}$ with $n s(L)>0$. Then, the following assertions hold.

1. Let $i \in[r]$. Then,

$$
\psi(i, j)=\left\{\begin{aligned}
-\prod_{h \in S} \partial_{h}(i), & \text { if } j \in S \Delta \mathcal{D}_{L}^{-}(S, i), \\
\prod_{h \in S} \partial_{h}(i), & \text { otherwise. }
\end{aligned}\right.
$$

2. Let $j \in[n]$. Then,

$$
\psi(i, j)=\left\{\begin{array}{cl}
-\prod_{h \in S} \partial_{h}(j), & \text { if } i \in(S \cap[r]) \Delta \mathcal{D}_{L}^{+}(S, i), \\
\prod_{h \in S} \partial_{h}(j), & \text { otherwise. }
\end{array}\right.
$$

3. Let $i \in[r]$. The ith row of the pseudococyclic matrix $M_{\psi}$ is uniformly signed if and only if one of the following two conditions hold.

(a) $S \Delta \mathcal{D}_{L}^{-}(S, i)=\varnothing$, in whose case, $\psi(i, j)=\prod_{h \in S} \partial_{h}(i)$, for all $j \leq n$.

(b) $S \Delta \mathcal{D}_{L}^{-}(S, i)=[n]$, in whose case, $\psi(i, j)=-\prod_{h \in S} \partial_{h}(i)$, for all $j \leq n$.

4. Let $j \in[n]$. The $j$ th column of the pseudocyclic matrix $M_{\psi}$ is uniformly signed if and only if one the following two conditions hold.

(a) $\quad(S \cap[r]) \Delta \mathcal{D}_{L}^{+}(S, i)=\varnothing$, in which case $\psi(i, j)=\prod_{h \in S} \partial_{h}(j)$ for all $i \leq r$.

(b) $\quad(S \cap[r]) \Delta \mathcal{D}_{L}^{+}(S, i)=[r]$, in which case $\psi(i, j)=-\prod_{h \in S} \partial_{h}(j)$ for all $i \leq r$.

Proof. The first two assertions and both sufficient conditions of the last two assertions follow readily from the definition (6). So, let us focus on the necessary condition of the third statement (that one of the last statements follows similarly). Thus, let us suppose the existence of a positive integer $i \leq r$ such that the $i$ th row of the pseudococyclic matrix $M_{\psi}$ is uniformly signed. Then, the mentioned definition (6) implies that either $\prod_{h \in S} \partial_{h}(j)=\prod_{h \in S} \partial_{h}(L[i, j])$ or $\prod_{h \in S} \partial_{h}(j)=-\prod_{h \in S} \partial_{h}(L[i, j])$ for all $j \leq n$. In the first case, $j \in S$ if and only if $L[i, j] \in S$, and hence, Condition (3a) holds. In the second case, $j \in S$ if and only if $L[i, j] \notin S$, and hence, Condition (3b) holds. In any case, the result follows then from the Latin rectangle condition of no repetitions of symbols in each row.

Example 10. Let $L$ be the Latin rectangle described in Example 1. The third assertion of Lemma 6 explains, for instance, the uniformity of signs of the first row of the pseudococyclic matrix $M_{\psi_{L ; 3} \psi_{L ; 4}}$ appearing in Example 6. More specifically, if we consider the subset $S=\{3,4\}$, then $\mathcal{D}_{L}^{-}(S, 1)=S$. That is, the the first row of $L$ satisfies the condition described in Lemma 6. (3a).

The third assertion of Lemma 6 also implies that $S=[4]$ is the only way to get a pseudococyclic matrix related to a pseudococycle $\psi=\prod_{h \in S \subseteq[4]} \psi_{L ; h}$ of $L$, whose rows are all uniformly signed. In such a case, all the signs within $M_{\psi}$ are negative.

Further, the fourth assertion of Lemma 6 explains, for instance, the uniformly signed columns of the following two pseudococyclic matrices.

$$
M_{\psi_{L ; 1} \psi_{L ; 2}} \equiv \begin{array}{|c|c|c|c|}
\hline- & - & - & - \\
\hline+ & - & + & - \\
\hline
\end{array}
$$

$$
M_{\psi_{L ; 2} \psi_{L ; 3}} \equiv \begin{array}{|c|c|c|c|}
\hline+ & + & - & - \\
\hline+ & + & - & - \\
\hline
\end{array}
$$


Thus, concerning the first pseudococyclic matrix, we have that, if $S=\{1,2\}$, then $\mathcal{D}_{L}^{+}(S, 2)=$ $S$ and $\mathcal{D}_{L}^{+}(S, 4)=\varnothing$. Concerning the second pseudococyclic matrix, we have that, if $S=$ $\{2,3\}$, then $\mathcal{D}_{L}^{+}(S, 1)=\mathcal{D}_{L}^{+}(S, 3)=\{2\}=S \cap[2]$ and $(S \cap[2]) \cap \mathcal{D}_{L}^{+}(S, 2)=(S \cap[2]) \cap$ $\mathcal{D}_{L}^{+}(S, 3)=\varnothing$.

Finally, the last statement of the fourth assertion of Lemma 6 explains, for instance, the uniformity of signs of the second and fourth columns of the pseudococyclic matrix $M_{\psi_{L ; 3}} M_{\psi_{L ; 4}}$, which is described in Example 6. Here, if $S=\{3,4\}$, then $\mathcal{D}_{L}^{+}(S, 2)=\varnothing$ and $\mathcal{D}_{L}^{+}(S, 4)=\{1,2\}$.

The following result characterizes the Latin rectangles $L \in \mathcal{R}_{r, n}$ with $r>1$ and $n s(L)>0$ over which a pseudococycle $\prod_{h \in S \subseteq \mathcal{H}(L)} \psi_{L ; h}$ exists, so that its related pseudococyclic matrix is partial Hadamard.

Proposition 7. Let $\psi=\prod_{h \in S \subseteq \mathcal{H}(L)} \psi_{L ; h}$ be a pseudococycle over a Latin rectangle $L \in \mathcal{R}_{r, n}$ with $r>1$ and $n s(L)>0$. The pseudococyclic matrix $M_{\psi}$ is partial Hadamard if and only if, for each pair of distinct positive integers $i_{1}, i_{2} \leq r$,

$$
\left|\mathcal{D}_{L}^{-}\left(S, i_{1}\right) \Delta \mathcal{D}_{L}^{-}\left(S, i_{2}\right)\right|=\frac{n}{2}
$$

Proof. Since the pseudococyclic matrix $M_{\psi}$ is partial Hadamard, all its rows are pairwise orthogonal and, hence, $\sum_{j \leq n} \psi\left(i_{1}, j\right) \psi\left(i_{2}, j\right)=0$. Then, the first statement of Lemma 6 implies that

$$
\left|\left(S \Delta \mathcal{D}_{L}^{-}\left(S, i_{1}\right)\right) \cap\left(S \Delta \mathcal{D}_{L}^{-}\left(S, i_{2}\right)\right)\right|+\left|\left([n] \backslash\left(S \Delta \mathcal{D}_{L}^{-}\left(S, i_{1}\right)\right)\right) \cap\left([n] \backslash\left(S \Delta \mathcal{D}_{L}^{-}\left(S, i_{2}\right)\right)\right)\right|=\frac{n}{2} .
$$

Equivalently, after all the set operations are done and simplified, we have that

$$
\left|[n] \backslash\left(\mathcal{D}_{L}^{-}\left(S, i_{1}\right) \Delta \mathcal{D}_{L}^{-}\left(S, i_{2}\right)\right)\right|=\frac{n}{2}
$$

and the result follows straightforwardly.

The worst-case complexity of the implicit algorithm described in Proposition 7 corresponds to a Latin square of order $n$. Thus, the time complexity of this algorithm is $\mathcal{O}\left(n^{4}\right)$, which is required for the computation of all the difference sets under consideration (notice that the computation of all the sets $\mathcal{D}_{L}^{-}(S, i)$ with $i \leq n$ only requires a time complexity of $\left.\mathcal{O}\left(n^{3}\right)\right)$.

Example 11. Let $L$ be the Latin rectangle defined in Example 1, and let us consider the pseudococyclic matrix associated with the pseudococycle $M_{\psi_{L ; 2}} M_{\psi_{L ; 4}}$, which is partial Hadamard.

$$
M_{\psi_{L ; 2}} M_{\psi_{L ; 4}} \equiv \begin{array}{|c|c|c|c|}
\hline+ & + & - & - \\
\hline- & + & + & - \\
\hline
\end{array}
$$

If we consider the subset $S=\{2,4\}$, then we have that $\mathcal{D}_{L}^{-}(S, 1)=\{2,3\}$ and $\mathcal{D}_{L}^{-}(S, 2)=$ $\{3,4\}$. Hence, $\mathcal{D}_{L}^{-}(S, 1) \Delta \mathcal{D}_{L}^{-}(S, 2)=\{2,4\}$, which is formed by two elements, as is required by Proposition 7.

Proposition 7 establishes a lower bound of the cardinality of $\mathcal{H}(L)$ for any $r \times n$ Latin rectangle $L$ with $r>1$ over which a pseudococyclic partial Hadamard matrix associated with the trivial cocycle exists.

Theorem 1. Let $\psi=\prod_{h \in S \subseteq \mathcal{H}(L)} \psi_{L ; h}$ be a pseudococycle over a Latin rectangle $L \in \mathcal{R}_{r, n}$ with $r>1$ and $n s(L)>0$ such that $|\mathcal{H}(L)|<\frac{n}{4}$. Then, the pseudococyclic matrix $M_{\psi}$ is not partial Hadamard. 
Proof. For each positive integer $i \leq r$, we have already indicated that $\left|\mathcal{D}_{L}^{-}(S, i)\right|=|S|$. As a consequence, $\left|\mathcal{D}_{L}^{-}\left(S, i_{1}\right) \Delta \mathcal{D}_{L}^{-}\left(S, i_{2}\right)\right| \leq 2 \cdot|S| \leq 2 \cdot|\mathcal{H}(L)|<\frac{n}{2}$ for all $i_{1}, i_{2} \leq r$. Then, the result follows straightforwardly from Proposition 7.

The following example illustrates how the bound described in Theorem 1 does not constitute a necessary condition for ensuring the non-existence of pseudococyclic partial Hadamard matrices associated with the trivial cocycle.

Example 12. Let us consider the following $2 \times 12$ Latin rectangle.

$L \equiv$\begin{tabular}{|c|c|c|c|c|c|c|c|c|c|c|c|}
\hline 1 & 2 & 4 & 3 & 5 & 6 & 7 & 8 & 9 & 10 & 11 & 12 \\
\hline 2 & 4 & 3 & 1 & 6 & 5 & 8 & 7 & 10 & 9 & 12 & 11 \\
\hline
\end{tabular}

It is easily verified that $N S(L)=\{(1,1,3),(1,1,4),(1,2,2),(1,2,3),(2,1,3),(2,1,4)\}$, and $\mathcal{H}(L)=\{1,3,4\}$. In addition, we have that

$$
\begin{array}{ll}
\mathcal{D}_{L}^{-}(\{1\}, 1) \Delta \mathcal{D}_{L}^{-}(\{1\}, 2)=\{1,4\}, & \mathcal{D}_{L}^{-}(\{3\}, 1) \Delta \mathcal{D}_{L}^{-}(\{3\}, 2)=\{3,4\}, \\
\mathcal{D}_{L}^{-}(\{4\}, 1) \Delta \mathcal{D}_{L}^{-}(\{4\}, 2)=\{2,3\}, & \mathcal{D}_{L}^{-}(\{1,3\}, 1) \Delta \mathcal{D}_{L}^{-}(\{1,3\}, 2)=\{1,3\}, \\
\mathcal{D}_{L}^{-}(\{1,4\}, 1) \Delta \mathcal{D}_{L}^{-}(\{1,4\}, 2)=\{1,2,3,4\}, & \mathcal{D}_{L}^{-}(\{3,4\}, 1) \Delta \mathcal{D}_{L}^{-}(\{3,4\}, 2)=\{2,4\}
\end{array}
$$

and $\mathcal{D}_{L}^{-}(\{1,3,4\}, 1) \Delta \mathcal{D}_{L}^{-}(\{1,3,4\}, 2)=\{1,2\}$. Thus, $\left|\mathcal{D}_{L}^{-}(S, 1) \Delta \mathcal{D}_{L}^{-}(S, 2)\right| \neq 6$ for all $S \subseteq$ $\mathcal{H}(L)$, and hence, no pseudococyclic partial Hadamard matrix associated with the trivial cocycle over L exists.

The next result deals with the pseudococyclic partial Hadamard matrices that have a uniformly signed row.

Theorem 2. Let $\psi=\prod_{h \in S \subseteq \mathcal{H}(L)} \psi_{L ; h}$ be a pseudococycle over a Latin rectangle $L \in \mathcal{R}_{r, n}$ with $n s(L)>0$ so that the pseudococyclic matrix $M_{\psi}$ is partial Hadamard. Then, there exists at most one positive integer $i \leq r$ such that $S \Delta \mathcal{D}_{L}^{-}(S, i) \in\{\varnothing$, [n] $\}$. If it exists, then $\left|S \Delta \mathcal{D}_{L}^{-}\left(S, i^{\prime}\right)\right|=\frac{n}{2}$ for all $i^{\prime} \in[r] \backslash\{i\}$. Moreover, $\frac{n}{4} \leq|S| \leq \frac{3 n}{4}$, and hence, $|\mathcal{H}(L)| \geq \frac{n}{4}$.

Proof. Since the partial Hadamard matrix $M_{\psi}$ can only have at most one uniformly signed row, the third statement of Lemma 6 implies the existence of at most one positive integer $i \leq r$ such that $S \Delta \mathcal{D}_{L}^{-}(S, i) \in\{\varnothing,[n]\}$. Thus, $\mathcal{D}_{L}^{-}(S, i) \in\{S,[n] \backslash S\}$. Now, let us consider a positive integer $i^{\prime} \in[r] \backslash\{i\}$. The following two cases arise.

- If $\mathcal{D}_{L}^{-}(S, i)=S$, then Proposition 7 implies that

$$
\frac{n}{2}=\left|\mathcal{D}_{L}^{-}(S, i) \Delta \mathcal{D}_{L}^{-}\left(S, i^{\prime}\right)\right|=\left|S \Delta \mathcal{D}_{L}^{-}\left(S, i^{\prime}\right)\right| .
$$

- If $\mathcal{D}_{L}^{-}(S, i)=[n] \backslash S$, then Proposition 7 implies that

$$
\frac{n}{2}=\left|\mathcal{D}_{L}^{-}(S, i) \Delta \mathcal{D}_{L}^{-}\left(S, i^{\prime}\right)\right|=\left|([n] \backslash S) \Delta \mathcal{D}_{L}^{-}\left(S, i^{\prime}\right)\right|=n-\left|S \Delta \mathcal{D}_{L}^{-}\left(S, i^{\prime}\right)\right| .
$$

Hence, $\left|S \Delta \mathcal{D}_{L}^{-}\left(S, i^{\prime}\right)\right|=\frac{n}{2}$. The rest of the result follows easily from the fact that the Latin rectangle condition of no repetitions of symbols per row implies that $\left|\mathcal{D}_{L}^{-}(S, i)\right|=|S|$.

The worst-case complexity of the implicit algorithm in Theorem 2 corresponds to a Latin square of order $n$. Thus, the time complexity of this algorithm is $\mathcal{O}\left(n^{3}\right)$, which is required for computing all the sets $\mathcal{D}_{L}^{-}(S, i)$ with $i \leq n$, and also for computing all the difference sets under consideration.

The following example illustrates the sharpness of both bounds concerning the cardinality of the subset $S$ in Theorem 2 .

Example 13. Let $L$ be the Latin rectangle defined in Example 1. In order to illustrate that the upper bound described in Theorem 2 is sharp, it is enough to consider the pseudococyclic matrix over $L$ 


$$
M_{\psi_{L ; 1} \psi_{L ; 2} \psi_{L ; 3}} \equiv \begin{array}{|c|c|c|c|}
\hline- & - & + & + \\
\hline- & - & - & - \\
\hline
\end{array}
$$

Thus, if we consider the subset $S=\{1,2,3\}$, then $\mathcal{D}_{L}^{-}(S, 2)=S$, and hence, $S \Delta \mathcal{D}_{L}^{-}(S, 2)=$ $\varnothing$. In addition, $\mathcal{D}_{L}^{-}(S, 1)=\{1,2,4\}$, and thus, $S \Delta \mathcal{D}_{L}^{-}(S, 1)=\{3,4\}$. That is, $\left|S \Delta \mathcal{D}_{L}^{-}(S, 1)\right|=2$.

Now, in order to illustrate the sharpness of the lower bound described in Theorem 2, we can make use of any of the $h$-pseudocoboundary matrices over $L$ with $h \in\{1,2,4\}$ that are described in Example 3. Thus, for instance, if we consider $S=\{1\}$, then $\mathcal{D}_{L}^{-}(S, 1)=S$ and $S \Delta \mathcal{D}_{L}^{-}(S, 2)=\{1,2\}$.

In order to illustrate the sharpness of this lower bound, but now avoiding the purely pseudocoboundary framework, let us consider the following $2 \times 8$ Latin rectangle.

$$
L^{\prime} \equiv \begin{array}{|l|l|l|l|l|l|l|l|}
\hline 1 & 2 & 3 & 4 & 5 & 6 & 7 & 8 \\
\hline 4 & 3 & 1 & 2 & 6 & 5 & 8 & 7 \\
\hline
\end{array}
$$

It is easily verified that $N S\left(L^{\prime}\right)=\{(1,1,3),(1,1,4),(1,2,1),(1,2,2)\}$, and $\mathcal{H}\left(L^{\prime}\right)=$ $\{3,4\}$. If we consider the subset $S=\{3,4\}$, then we have that $\mathcal{D}_{L^{\prime}}^{-}(S, 1)=S$ and $S \Delta \mathcal{D}_{L^{\prime}}^{-}(S, 2)=$ $\{1,2,3,4\}$. Then, the pseudococyclic matrix over $L^{\prime}$ associated with the pseudococycle $\psi_{L^{\prime} ; 3} \psi_{L^{\prime} ; 4}$ is partial Hadamard.

$$
M_{\psi_{L^{\prime} ; 3} \psi_{L^{\prime} ; 4}} \equiv \begin{array}{|c|c|c|c|c|c|c|c|}
\hline+ & + & + & + & + & + & + & + \\
\hline- & - & - & - & + & + & + & + \\
\hline
\end{array}
$$

\section{Pseudococyclic Partial Hadamard Matrices Associated with Non-Trivial Cocycles}

Let us finish our study by focusing on the pseudococycles $\prod_{h \in S \subseteq \mathcal{H}(L)} \psi_{L ; h} \phi$ over a given Latin rectangle $L \in \mathcal{R}_{r, n}$ with $n s(L)>0$ where the cocycle $\phi$ over $L$ is not trivial. The following result generalizes Proposition 7 by characterizing the Latin rectangles over which one such pseudococycle exists so that its related pseudococyclic matrix is partial Hadamard.

Proposition 8. Let $\psi=\prod_{h \in S \subseteq \mathcal{H}(L)} \psi_{L ; h} \phi$ be a pseudococycle over a Latin rectangle $L \in \mathcal{R}_{r, n}$ with $n s(L)>0$ for some cocycle $\phi$ over $L$. The pseudococyclic matrix $M_{\psi}$ is partial Hadamard if and only if, for each pair of positive integers $i_{1}, i_{2} \leq r$, the set

$$
\left\{j \in \mathcal{D}_{L}^{-}\left(S, i_{1}\right) \Delta \mathcal{D}_{L}^{-}\left(S, i_{2}\right): \phi\left(i_{1}, j\right)=\phi\left(i_{2}, j\right)\right\} \cup\left\{j \notin \mathcal{D}_{L}^{-}\left(S, i_{1}\right) \Delta \mathcal{D}_{L}^{-}\left(S, i_{2}\right): \phi\left(i_{1}, j\right)=-\phi\left(i_{2}, j\right)\right\}
$$

has cardinality $\frac{n}{2}$.

Proof. The result follows in a similar way to the proof of Proposition 7, once it is observed that $\sum_{j \leq n} \psi\left(i_{1}, j\right) \psi\left(i_{2}, j\right)=0$ if and only if

$$
\sum_{j \in \mathcal{D}_{L}^{-}\left(S, i_{1}\right) \Delta \mathcal{D}_{L}^{-}\left(S, i_{2}\right)} \phi\left(i_{1}, j\right) \phi\left(i_{2}, j\right)=\sum_{j \notin \mathcal{D}_{L}^{-}\left(S, i_{1}\right) \Delta \mathcal{D}_{L}^{-}\left(S, i_{2}\right)} \phi\left(i_{1}, j\right) \phi\left(i_{2}, j\right) .
$$

Keeping in mind the observation made just after Proposition 7, the time complexity of the implicit algorithm described in Proposition 8 is $\mathcal{O}\left(n^{5}\right)$, which corresponds once more to the Latin square case.

Example 14. Let $L$ be the Latin rectangle defined in Example 1 and let us consider the pseudococyclic matrix $M_{\psi_{L_{2} ;} \psi_{L_{2} ; 4}} \phi_{2}$ described in Example 6, which is not partial Hadamard. If we again take $S=\{3,4\}$, then we have that $\mathcal{D}_{L}^{-}(S, 1)=\{3,4\}$ and $\mathcal{D}_{L}^{-}(S, 2)=\{1,4\}$. Hence, $\mathcal{D}_{L}^{-}(S, 1) \Delta \mathcal{D}_{L}^{-}(S, 2)=\{1,3\}$. In this case, the set defined in (7) concerning the pseudococycle $\psi_{L ; 3} \psi_{L ; 4} \phi_{2}$ is the set $\{2,3,4\}$, which is not formed by two elements, as is required by Proposition 8. 
Example 15. Let us consider the Latin rectangle $L \in \mathcal{R}_{2,12}$ that is described in Example 12, and let us define the cocycle $\phi$ over $L$ that is represented by the matrix

$M_{\phi} \equiv$\begin{tabular}{|c|c|c|c|c|c|c|c|c|c|c|c|}
\hline+ & + & + & + & + & + & + & + & + & + & + & + \\
\hline+ & + & + & + & - & - & + & + & + & + & + & + \\
\hline+ & + & + & + & + & + & + & + & + & + & + & + \\
\hline
\end{tabular}

Notice here that the third row of this cocyclic matrix corresponds to the positive integer $4 \in \mathcal{S}(L)=\{1,2,4\}$. The pseudococyclic matrix associated with the pseudococycle $\psi_{L ; 1} \psi_{L ; 4} \phi$ is

$$
M_{\psi_{L ; 1} \psi_{L ;} \phi} \equiv \begin{array}{|c|c|c|c|c|c|c|c|c|c|c|c|}
\hline- & - & + & + & - & - & - & - & - & - & - & - \\
\hline- & - & + & + & - & - & + & + & + & + & + & + \\
\hline
\end{array}
$$

which is partial Hadamard. If we consider the subset $S=\{1,4\}$, then we have that $\mathcal{D}_{L}^{-}(S, 1) \Delta \mathcal{D}_{L}^{-}$ $(S, 2)=[4]$. Moreover, the set defined in (7) concerning the pseudococycle $\psi_{L ; 1} \psi_{L ; 4} \phi$ is the set $\{1,2,3,4,5,6\}$, which is formed by six elements, as is required by Proposition 8.

The pseudococyclic partial Hadamard matrix in Example 15 shows that Theorem 1 cannot be generalized for pseudococycles associated with non-trivial cocycles. Concerning the possible generalization of Theorem 2, the following result deals with the case of a pseudocycle related to a non-trivial cocycle whose pseudococyclic partial Hadamard matrix contains a uniformly signed row.

Proposition 9. Let $\psi=\prod_{h \in S \subseteq \mathcal{H}(L)} \psi_{L ; h} \phi$ be a pseudococycle over a Latin rectangle $L \in \mathcal{R}_{r, n}$ with $n s(L)>0$ for some cocycle $\phi$ over $L$. If the pseudococyclic matrix $M_{\psi}$ is partial Hadamard, then there exists at most one positive integer $i \leq r$ and an integer $a \in\{-1,1\}$ such that

$$
\phi(i, j)=\left\{\begin{aligned}
a, & \text { if } j \in S \Delta \mathcal{D}_{L}^{-}(S, i), \\
-a, & \text { if } j \notin S \Delta \mathcal{D}_{L}^{-}(S, i) .
\end{aligned}\right.
$$

Proof. Since the pseudococyclic matrix $M_{\psi}$ is partial Hadamard, it can only have at most one uniformly signed row. Hence, there exists at most one positive integer $i \leq r$ such that $\psi\left(i, j_{1}\right)=\psi\left(i, j_{2}\right)$ for all pairs of positive integers $j_{1}, j_{2} \leq n$. The result then follows from the third statement of Lemma 6, together with the definition of the cocycle $\phi$.

Example 16. Let us consider the Latin rectangle $L \in \mathcal{R}_{2,12}$ that is described in Example 12 and let us consider the cocycle $\phi$ over $L$ that is represented by the matrix

$$
M_{\phi} \equiv \begin{array}{|c|c|c|c|c|c|c|c|c|c|c|c|}
\hline- & - & + & + & - & - & - & - & - & - & - & - \\
\hline+ & + & - & - & - & - & - & - & - & - & - & - \\
\hline+ & + & + & + & + & + & + & + & + & + & + & + \\
\hline
\end{array}
$$

Further, let us consider the subset $S=\{1,4\}$. Then, $S \Delta \mathcal{D}_{L}^{-}(S, 1)=\{3,4\}$ and $S \Delta \mathcal{D}_{L}^{-}(S, 2)=$ $\{1,2\}$. According to Proposition 9, the pseudococyclic matrix $M_{\psi_{L ; 1} \psi_{L ; 4} \phi}$ over $L$ is not partial Hadamard. In fact,

$$
M_{\psi_{L ; 1} \psi_{L ;} \phi} \equiv \begin{array}{|c|c|c|c|c|c|c|c|c|c|c|c|}
\hline+ & + & + & + & + & + & + & + & + & + & + & + \\
\hline- & - & - & - & - & - & - & - & - & - & - & - \\
\hline
\end{array} .
$$

\section{Conclusions and Further Work}

In this paper, we have introduced the concepts of both the pseudocoboundary and pseudococycle over a Latin rectangle (see Section 3) as a natural generalization of the similar notions recently described in [17] over quasigroups. To this end, we have made use of the cocyclic framework over Latin rectangles previously introduced by the authors in [18]. Both cocyclic and pseudococyclic developments over Latin rectangles together 
constitute a much more general framework than the classical cocyclic framework over groups. Its potential has already been illustrated in the mentioned papers by means of examples of (pseudo)cocyclic Hadamard matrices over quasigroups that are not cocyclic over any group. This paper constitutes a step forward in this regard. Thus, for instance, Example 5 illustrates a pseudocoboundary Hadamard matrix over a Latin square that is not cocyclic over any Latin rectangle.

Let us remark that this paper is conceived as an introductory stage concerning the fundamentals of both the pseudocoboundary and the pseudococyclic frameworks over Latin rectangles. Particularly, in Section 4, we completely answered both Problems 1 and 2 concerning the conditions under which we may ensure either the existence of a partial Hadamard matrix that is a pseudocoboundary over a given Latin rectangle or, reciprocally, the existence of a Latin rectangle over which a given partial Hadamard matrix is a pseudocoboundary. More specifically, Propositions 1 and 2 give the answer of the first question, whereas the second one is answered by Lemma 1, together with Propositions 4-6.

Furthermore, we have also dealt with the problem of determining under which conditions we may ensure the existence of a partial Hadamard matrix that is pseudococyclic over a given Latin rectangle. To this end, we have distinguished two distinct frameworks (see Sections 5 and 6), depending on whether we make use of trivial cocycles or not. The reciprocal problem concerning the conditions under which we may ensure the existence of a Latin rectangle over which a given partial Hadamard matrix is pseudococyclic is established as future work. Once this last question is solved, the next natural stage would be the construction of pseudococyclic partial Hadamard matrices of higher dimensions in order to deal with the Hadamard conjecture described in the introductory section, which indeed constitutes the keystone of the theory of Hadamard matrices.

The following open questions are also established as future work. They generalize similar ones described for the cocyclic development of Hadamard matrices over Latin rectangles [18].

Problem 3. Let $M$ be an $r \times n$ partial Hadamard matrix that is not pseudococyclic over any Latin rectangle. Does there exist, however, a partial Hadamard equivalent matrix in the same equivalence class of $M$ for which one such Latin rectangle can be found?

Problem 4. Let us consider an equivalence class of Hadamard matrices such that none of them are cocyclic over any finite group. Does there exist, however, a Hadamard matrix within such a class that is pseudococyclic over a Latin rectangle?

Pseudococycles over Latin rectangles have been introduced in this paper as the product of a cocycle with some pseudocoboundaries. A possible generalization of this notion consists of enabling the product of a cocycle not only with pseudocoboundaries (related to non-associative triples), but also with elementary coboundaries (related to associative triples). This would constitute a more general framework that puts together both cocyclic and pseudococyclic frameworks over Latin rectangles. Its formal description and characterization is also proposed as future work.

Finally, another question to take into consideration for further study is the following one. Both the pseudocoboundary and the pseudococyclic frameworks over Latin rectangles described in this paper are based on the existence of non-associative triples within a Latin rectangle. As was already indicated in the introductory section and in Section 3, the study of this type of triple in the case of dealing with Latin squares has received particular attention in the recent literature [32-36] because of its possible application in different areas as cryptography [30,31]. A comprehensive study of non-associative triples in the case of dealing with Latin rectangles instead of Latin squares is established, therefore, as natural further work. 
Author Contributions: Conceptualization, V.Á., R.M.F. and F.G.; Data curation, M.D.F., F.G. and M.B.G.; Formal analysis, V.Á., R.M.F. and F.G.; Investigation, V.Á. and R.M.F.; Methodology, R.M.F., M.D.F. and M.B.G.; Software, V.Á. and R.M.F.; Supervision, F.G.; Validation, M.D.F. and M.B.G.; Writing—original draft, R.M.Falcón; Writing—review \& editing, V.Á., R.M.F., M.D.F. and F.G. All authors have read and agreed to the published version of the manuscript.

Funding: This research received no external funding.

Institutional Review Board Statement: Not applicable.

Informed Consent Statement: Not applicable.

Acknowledgments: This work was partially supported by the Research Project FQM-016 from Junta de Andalucía. In addition, the authors want to express their gratitude to the anonymous referees for the comprehensive reading of the paper and their pertinent comments and suggestions, which helped improve the manuscript. Particularly, we are grateful to the anonymous referee who suggested to us the more general framework described in the conclusion section concerning the product of a cocycle with both types of pseudocoboundaries and elementary coboundaries of a given Latin rectangle.

Conflicts of Interest: The authors declare no conflict of interest.

\section{Appendix A. Glossary of Symbols}

$\mathcal{H}(L) \quad$ The set of positive integers satisfying Condition (5).

$M_{\psi_{L ; h}}$ The $h$-pseudocoboundary matrix associated to a Latin rectangle $L \in \mathcal{R}_{r, n}$, with $h \in \mathcal{H}(L)$.

$[n] \quad$ The set $\{1, \ldots, n\}$.

$n s(L) \quad$ The non-associative index of a Latin rectangle $L$.

$N S(L)$ The set of non-associative triples of a Latin rectangle $L$.

$\mathcal{R}_{r, n} \quad$ The set of $r \times n$ Latin rectangles with entries in $[n]$.

$\mathcal{S}(L) \quad$ The subset of symbols describing the rows of a cocyclic matrix over a Latin rectangle $L$.

$\psi_{L ; h} \quad$ The $h$-pseudocoboundary over a Latin rectangle $L$, with $h \in \mathcal{H}(L)$.

\section{References}

1. Paley, R.E.A.C. On orthogonal matrices. J. Math. Phys. 1933, 12, 311-320. [CrossRef]

2. Horadam, K.J. Hadamard Matrices and Their Applications; Princeton University Press: Princeton, NJ, USA, 2007.

3. Horadam, K.J.; de Launey, W. Cocyclic development of designs. J. Algebr. Comb. 1993, 2, 267-290; Erratum: J. Algebr. Comb. 1994, 3, 129. [CrossRef]

4. de Launey, W. On the construction of n-dimensional designs from 2-dimensional designs. Australas. J. Comb. 1990, 1, 67-81.

5. de Launey, W.; Horadam, K.J. A weak difference set construction for higher dimensional designs. Des. Codes Cryptogr. 1993, 3, 75-87. [CrossRef]

6. Horadam, K.J.; de Launey, W. Generation of cocyclic Hadamard matrices. Math. Appl. 1995, 325, $279-290$.

7. Sylvester, J.J. LX. Thoughts on inverse orthogonal matrices, simultaneous sign-successions, and tessellated pavements in two or more colours, with applications to Newton's rule, ornamental tile-work, and the theory of numbers. Lond. Edinb. Dublin Philos. Mag. J. Sci. 1867, 34, 461-475. [CrossRef]

8. Williamson, J. Hadamard's determinant theorem and the sum of four squares. Duke Math. J. 1944, 11, 65-81. [CrossRef]

9. Ito, N. On Hadamard groups III. Kyushu J. Math. 1997, 51, 369-379. [CrossRef]

10. de Launey, W.; Flannery, D.L.; Horadam, K.J. Cocyclic Hadamard matrices and difference sets. Discret. Appl. Math. 2000, 102, 47-61. [CrossRef]

11. de Launey, W.; Smith, M.J. Cocyclic orthogonal designs and the asymptotic existence of cocyclic Hadamard matrices and maximal size relative difference sets with forbidden subgroup of size 2. J. Comb. Theory Ser. A 2001, 93, 37-92. [CrossRef]

12. Catháin, P. Group Actions on Hadamard Matrices. Master's Thesis, National University of Ireland, Galway, Ireland, 2008.

13. de Launey, W.; Flannery, D. Algebraic Design Theory; American Mathematical Society: Providence, RI, USA, 2011.

14. Egan, R.; Flannery, D.L. Automorphisms of generalized Sylvester Hadamard matrices. Discret. Math. 2017, 340, 516-523. [CrossRef]

15. Fletcher, R.J.; Gysin, M.; Seberry, J. Application of the discrete Fourier transform to the search for generalised Legendre pairs and Hadamard matrices. Australas. J. Comb. 2001, 23, 75-86.

16. Goethals, J.M.; Seidel, J.J. Orthogonal matrices with zero diagonal. Can. J. Math. 1967, 19, 1001-1010. [CrossRef]

17. Álvarez, V.; Armario, J.A.; Falcón, R.M.; Frau, M.D.; Gudiel, F.; Güemes, M.B.; Osuna, A. On cocyclic Hadamard matrices over Goethals-Seidel loops. Mathematics 2020, 8, 1-22. [CrossRef]

18. Álvarez, V.; Falcón, R.M.; Frau, M.D.; Gudiel, F.; Güemes, M.B. Cocyclic Hadamard matrices over Latin rectangles. Eur. J. Comb. 2019, 79, 74-96. [CrossRef] 
19. Craigen, R.; Faucher, G.; Low, R.; Wares, T. Circulant partial Hadamard matrices. Linear Algebra Appl. 2013 , 439, $3307-3317$. [CrossRef]

20. Kao, M.H. Universally optimal fMRI designs for comparing hemodynamic response functions. Stat. Sin. 2015, 25, 499-506. [CrossRef]

21. Shi, F; Zhang, X.; Guo, Y. Constructions of unextendible entangled bases. Quantum Inf. Process. 2019, 18, 14.

22. Banica, T.; Skalski, A. The quantum algebra of partial Hadamard matrices. Linear Algebra Appl. 2015, 469, 364-380. [CrossRef]

23. Lin, Y.L.; Phoa, F.K.H.; Kao, M.H. Circulant partial Hadamard matrices: construction via general difference sets and its application to fMRI experiments. Stat. Sin. 2017, 27, 1715-1724.

24. Banica, T.; Özteke, D.; Pittau, L. Isolated partial Hadamard matrices and related topics. Open Syst. Inf. Dyn. 2018, 25, 27. [CrossRef]

25. Álvarez, V.; Armario, J.A.; Falcón, R.M.; Frau, M.D.; Gudiel, F.; Güemes, M.B.; Osuna, A. Generating binary partial Hadamard matrices. Discret. Appl. Math. 2019, 263, 2-7. [CrossRef]

26. Boucetta, C.; Nour, B.; Moungla, H.; Lahlou, L. An IoT scheduling and interference mitigation scheme in TSCH using Latin rectangles. In Proceedings of the 2019 IEEE Global Communications Conference (GLOBECOM), Waikoloa, HI, USA, 9-13 December 2019; pp. 1-6.

27. Chang, C. Reliable and Secure Storage with Erasure Codes for OpenStack Swift in PyECLib. Master's Thesis, KTH Royal Institute of Technology, Stockholm, Sweden, 2016.

28. Stones, R.J. K-plex 2-erasure codes and Blackburn partial Latin squares. IEEE Trans. Inform. Theory 2020, 66, 3704-3713 [CrossRef]

29. Gligoroski, D.; Kralevska, K. Expanded combinatorial designs as tool to model network slicing in 5G. IEEE Access 2019, 7, 54879-54887. [CrossRef]

30. Dénes, J.; Keedwell, A.D. A new authentication scheme based on Latin squares. Discret. Math. 1992, 106/107, 157-161. [CrossRef]

31. Grošek, O.; Horák, P. On quasigroups with few associative triples. Des. Codes Cryptogr. 2012, 64, 221-227. [CrossRef]

32. Drápal, A.; Lisoněk, P.. Maximal nonassociativity via nearfields. Finite Fields Appl. 2020, 62, 27. [CrossRef]

33. Artamonov, V.A.; Chakrabarti, S.; Pal, S.K. Characterizations of highly non-associative quasigroups and associative triples. Quasigroups Relat. Syst. 2017, 25, 1-19.

34. Drápal, A.; Valent, V. Few associative triples, isotopisms and groups. Des. Codes Cryptogr. 2018, 86, 555-568. [CrossRef]

35. Drápal, A.; Valent, V. High nonassociativity in order 8 and an associative index estimate. J. Comb. Des. 2019, 27, 205-228. [CrossRef]

36. Drápal, A.; Valent, V. Extreme nonassociativity in order nine and beyond. J. Comb. Des. 2020, 28, 33-48. [CrossRef]

37. Bruck, R.H. A Survey of Binary Systems; Springer: New York, NY, USA, 1958.

38. Moufang, R. Zur Struktur von Alternativkörpern. Math. Ann. 1935, 110, 416-430. [CrossRef]

39. Gavrilov, M.; Čobanov, I. The index of non-associativity of multiplicative structures. Annu. Univ. Sofia Fac. Sci. Phys. Math. Livre 1 Math. 1963, 56, 23-26.

40. Drápal, A.; Kepka, T. Sets of associative triples. Eur. J. Comb. 1985, 6, 227-231. [CrossRef]

41. Kepka, T.; Trch, M. Groupoids and the associative law. I. Associative triples. Acta Univ. Carol. Math. Phys. 1992, 33, 69-86.

42. Waldhauser, T. Almost associative operations generating a minimal clone. Discuss. Math. Gen. Algebra Appl. 2006, 26, 45-73. [CrossRef]

43. Climescu, A.C. Etudes sur la théorie des systèmes multiplicatifs uniformes I. L'indice de non-associativité. Bull. Ecole Polytech. Jassy 1947, 2, 347-371.

44. Ježek, J.; Kepka, T. Notes on the number of associative triples. Acta Univ. Carol. Math. Phys. 1990, 31, 15-19.

45. Drápal, A. On quasigroups rich in associative triples. Discret. Math. 1983, 44, 251-265. [CrossRef]

46. Dénes, J.; Keedwell, A.D. Latin Squares and Their Applications; Academic Press: New York, NY, USA; London, UK, 1974.

47. Drápal, A.; Kepka, T. Group distances of Latin squares. Comment. Math. Univ. Carol. 1985, 26, $275-283$. 